\title{
A Robust Face Recognition System in Image and Video
}

\author{
Alireza Tofighi \\ Department of Computer Engineering, Faculty of Engineering, University of Isfahan, Isfahan, 81746, Iran \\ Email: alirezatofighi.64@gmail.com \\ Nima Khairdoost \\ Department of Computer Engineering, Tabaran Institute of Higher Education, Mashhad, Iran \\ Email: n.khairdoost@gmail.com \\ S. Amirhassan Monadjemi, Associate Professor \\ Department of Computer Engineering, Faculty of Engineering, University of Isfahan, Isfahan, 81746, Iran \\ Email: monadjemi@eng.ui.ac.ir \\ Kamal Jamshidi, Associate Professor \\ Department of Computer Engineering, Faculty of Engineering, University of Isfahan, Isfahan, 81746, Iran \\ Email: jamshidi@eng.ui.ac.ir
}

\begin{abstract}
Face detection and recognition has always been one of the research interests to researchers in the field of the biometric identification of individuals. Problems such as environmental lighting, different skin color, complex background, etc affect on the detection and recognition of individuals. This paper proposes a method to enhance the performance of face detection and recognition systems. Our method, basically consists of two main parts: firstly, we detect faces and then recognize the detected faces. In the detection step, we use the skin color segmentation combined with AdaBoost algorithm, which is fast and also more accurate compared to the other known methods. Also, we use a series of morphological operators to improve the face detection performance. Recognition part consists of three steps: dimension reduction using Principal Component Analysis (PCA), feature selection using Linear Discriminant Analysis (LDA), and k-Nearest Neighbor (K-NN) or Support Vector Machine (SVM) based classification. Combination of PCA and LDA is used for improving the capability of LDA when a few samples of images are available. We test the system on the face databases. Experimental results show that the system is robust enough to detect faces in different lighting conditions, scales, poses, and skin colors from various races. Also, the system is able to recognize face with less misclassification compared to the previous methods.
\end{abstract}

Index Terms-Skin color segmentation, morphological operations, AdaBoost, PCA, LDA, K-NN, SVM.

\section{INTRODUCTION}

In general, a biometric system uses physiological characteristics (such as fingerprint, retina, iris, face, palms of hands) or behavioral characteristics (such as voice, handwriting, etc.) to identify or confirm a person's identity.

The face recognition system is one of the methods of biometric identification of individuals by face features [1] During the past decade, face recognition has become a common field of research in the field of machine vision and also one of the successful applications of image processing which plays an important role in many today applications. Among its applications we can note to the recognition individuals for law enforcement, security authentication for making access to the banking system, smart cards, surveillance systems, entertainment [2-4] and others.

In general, a face detection system consists of the following parts [5]:

-Detection face(s) from input video

-Feature extraction for determining the identity of face(s) in the video

-Comparison with images recorded in the database and identify the face(s)

Although feature extraction and classification modules, are two modules that cause more differentiation between the faces recognition systems, but the faces detection module has an essential role in the performance of face recognition system, so that correct and accurate face detection will increase efficiency and improve performance of face recognition unit. Therefore in this paper we try to consider problems such as changing the face angle, the brightness of the face, the facial, background of the images and the occlusion that are the limiting environmental factors of the work on face recognition and have a great effect on system performance, to give an appropriate solution in order to provide a reliable and efficient system. 
According to studies, face detection methods can be divided into four different categories [6]: (1) Knowledgebased methods that encode the human knowledge about the face into rules that express the relationship between facial features such as detection of a triangle in the face, (2) Feature-based methods that use features such as color, edge, and texture to locate faces even when the lighting condition, scale, or pose change, (3) Template matching methods that use patterns already stored, and decision for face detection comes from correlations between the stored patterns and the input image, and (4) Appearancebased methods, where unlike the template matching methods, patterns are learned by a set of training images to detect faces.

Since face detection is mostly used in video and realtime applications, its algorithms must be fast and computationally not complex as far as possible. Furthermore, they must be effective and accurate. So we proposed two scenarios that in first we employ a combination of skin color segmentation and AdaBoost in order to benefit from the advantages of both. And in second, using the Background Differencing method we isolate the foreground region and then apply the SkinColor algorithm on it and next, we take advantage of the AdaBoost algorithm.

Moreover, among various solutions to the face recognition [7], the most successful seems to be those methods which extract features from faces to optimally represent faces belong to a class and separate faces from different classes [8]. This means that prior to the recognition, the features should be extracted from faces. Recently, many methods have been proposed for feature extraction [4, 7-12]. The main trend in face feature extraction is to use only features having high separability power, while ignoring the rest and representing the data in a lower dimensional space, computed through a linear or non-linear transformation satisfying certain properties. Statistical techniques have been widely used to extract the features of the face patterns. Principal Component Analysis (PCA) [9-12], Linear Discriminant Analysis (LDA) [11, 13-16], Gabor filtering [8, 17-19], and Discrete Cosine Transform (DCT) [20-22] are the main techniques used for data reduction and feature extraction. PCA selects features which are effective in class representation, while LDA algorithm selects features that are most effective for class separability. According to a study in [23], we can see that PCA might outperform LDA when the number of samples per class is small, while in the case of training set with a large number of samples, the LDA still outperforms the PCA. It can be said that the computational cost of the LDA is much higher than the PCA method and PCA is less sensitive to different training data sets. However in some cases, simulations using the LDA method show higher performance compared to the PCA method [23]. We use a combination of PCA and LDA to exploit the advantages of both methods [11]. By applying PCA to preprocess face images, we get low dimensionality images which are ready to extract LDA features [11].

In order to classify the face images, there are different known classifiers such as K-nearest neighbor (KNN) [10], artificial neural networks (ANNs) $[8,11]$ and support vector machine (SVM) [9, 20, 24-27]. We employ PCA and LDA methods for feature selection and powerful SVM classifiers to classify face images, and a high recognition performance is expected.

The next section contains the description for the face detection method. Section III describes the PCA and LDA dimension reduction and feature selection algorithms and also the SVM methods which are used for face classification. Section IV describes the proposed method. The experimental results are presented in Section $\mathrm{V}$ and the conclusions are finally drawn in Section VI.

\section{FACE DETECTION USING SKIN-COLOR AND ADABOOST}

As mentioned, in this scenario we first use the Skincolor method to extract skin regions that are candidates to the face. To further improve the performance of face detection and to ensure higher accuracy, we used a series of morphological operations to improve the candidate regions. Next, the faces are detected using AdaBoost algorithm and the Haar-like features extracted from the candidate regions. This method is enough fast and suitable for video processing and uses the benefits of both Skin-color and AdaBoost methods. The above scenario is shown in Fig. 1.

\section{A. Skin Color Segmentation}

The skin plays an important role in many image processing applications including face detection, face tracking, motion analysis, and many different areas of interaction between humans and machines. Since the information based on skin color detection techniques are efficient in terms of computational and its processing is faster than many other face features and also it is robust to changes such as scale, occlusion, etc. it has attracted the attention of many researchers. Studies indicate that amongst the people with various skin colors, the main difference is in the brightness, and not the chrominance. Therefore, distribution of skin color clusters of faces with different skin color will be in a small area of the color space [6].

The main steps for detecting skin in an image using the color information include representing the image pixels in a suitable color space, modeling and classification of the skin and non-skin pixels using a suitable distribution. 


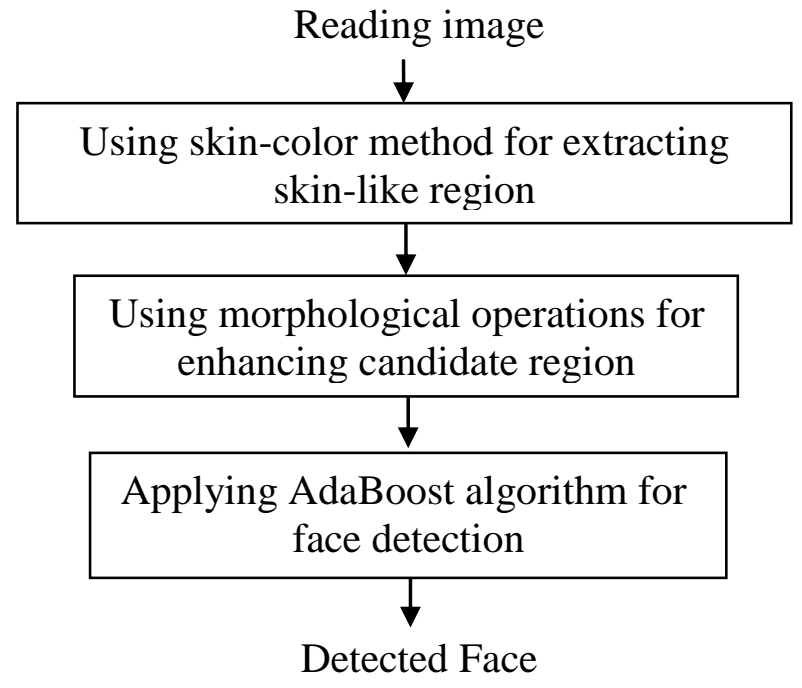

Fig. 1. Flowchart of the First Scenario

\section{Color Space}

Due to the variability and sensitivity of skin color to several factors such as lighting conditions, individual characteristics, ethnicity, camera characteristics, and so on [28], selection of a proper color space can have a great impact on the skin detection performance.

Studies on more than ten common color spaces in [29], indicate that one of the best color spaces for skin color detection is $\mathrm{YC}_{\mathrm{b}} \mathrm{C}_{\mathrm{r}}$. The conversion formula between the $\mathrm{YC}_{\mathrm{b}} \mathrm{C}_{\mathrm{r}}$ and $\mathrm{RGB}$ is as follows (1):

$$
\left\{\begin{array}{l}
Y=0.299 R-0.587 G-0.114 B \\
C_{b}=0.564 *(B-Y)+128 \\
C_{r}=0.713 *(R-Y)+128
\end{array}\right.
$$

Research has shown that skin colors differ more in their luminance compared to their chrominance. So it would be reasonable take away the luminance components for skin classification. The $\mathrm{YC}_{\mathrm{b}} \mathrm{C}_{\mathrm{r}}$ color space decomposes the RGB color into luminance and chrominance information. Due to the separation of chromaticity and illumination component in $\mathrm{YC}_{\mathrm{b}} \mathrm{C}_{\mathrm{r}}$ model, it is a good choice for skin color detection. Therefore, the color space employed for skin color segmentation is $\mathrm{YC}_{\mathrm{b}} \mathrm{C}_{\mathrm{r}}$.

In Fig. 2.b you can see an example of converting RGB image to the $\mathrm{YC}_{\mathrm{b}} \mathrm{C}_{\mathrm{r}}$ image.

\section{Skin Color Model}

After choosing the color space, we need to specify the skin color model to segment the skin and non-skin pixels. Different methods such as explicit skin-color space thresholding, histogram model, Gaussian classifiers, elliptical boundary model, MLP classifier, Maximum entropy classifier, and Bayesian network classifier [28] have been proposed to carry out that task.

One of the challenges in skin detection algorithms is their time complexity. For example, in methods such as nearest neighborhood, time order is about several seconds and sometimes a minute or more, which is much more than the Gaussian based scheme. Furthermore, Gaussian method needs learning and memory storage. Therefore, to improve the efficiency of the skin color segmentation, the thresholding model is applied.

As before, skin color of different people is placed in small region of color space. Thus we can use this issue and apply thresholding (2):

$$
\text { segment }=\left\{\begin{array}{cc}
1, & \text { if }\left(80 \leq C_{b} \leq 135 \text { and } 136 \leq C_{r} \leq 177\right) \\
0, & \text { otherwise }
\end{array}\right.
$$

Pixels that their values are in the range of specified threshold, are considered as skin pixels and marked as white, and others are considered as non-skin pixels and marked as black. By this process a binary image is produced. A sample result of applying the skin-color algorithm on a frame of a video is shown in Fig. 2.c.

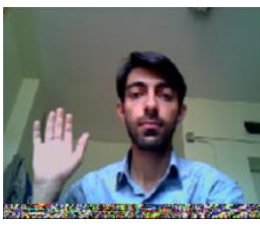

(a)

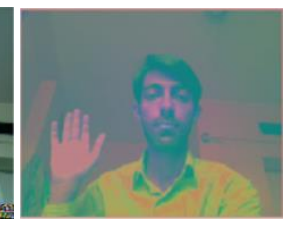

(b)

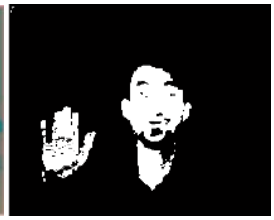

(c)
Fig. 2. Skin color segmentation. a)The original image, b) The image in $\mathrm{YC}_{\mathrm{b}} \mathrm{C}_{\mathrm{r}}$ space, $\mathrm{c}$ ) The binary image

\section{B. Binary Morphological Operations}

For better preparation of face region candidates, it is appropriate to use the morphological operations on the output of the previous step. Using morphological operations, we can eliminate holes that have been formed in some parts of the eye, mouth, and nose in the skin color segments. Therefore, by applying morphological operations on it, fewer regions mistakenly are considered as the face candidates and the use of it will generate fewer false alarms. So we employ the morphological operations such as erosion, dilation, and filling. Steps of applying morphological operators which were employed are shown in Fig. 3.

The input of this stage is the binary image from the Skin-color segmentation of the previous stage. Firstly, morphological opening operator is applied to remove the very small objects from the image, while keeps the shape and size of large objects in the image. The definition of a morphological opening of an image, is an erosion operation that is done after the dilation operation and both of them use same structural elements. For this purpose we use here circular structural element with radius 1.

In the following, operation of filling holes is done in order to maintain and keep the face parts together, and this is applied before the second larger opening operation. Because otherwise the resulting output from the image contains spaces, and holes in the faces.

Then morphological opening operation is done to remove the small to medium objects, which are certainly much smaller than a face. To do this operation, a circular structural element with a radius of 6 is employed. 
Fig. 4 shows the results of applying the morphological operations on the results of skin color segmentation.

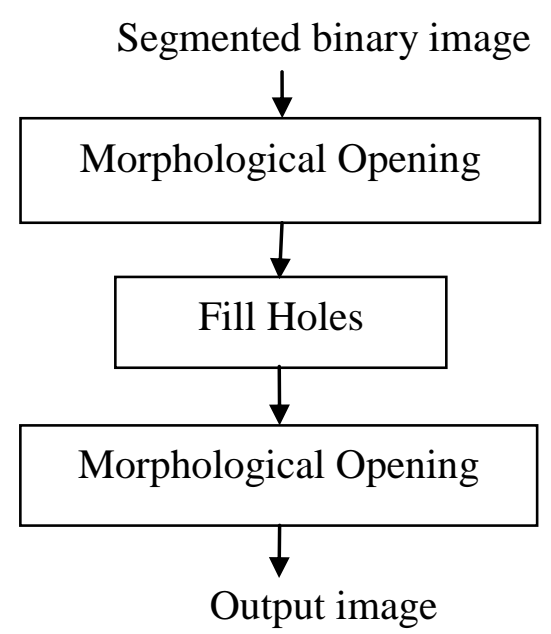

Fig. 3. The morphological processing on the segmented image

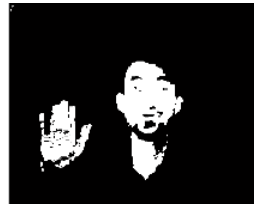

(b)

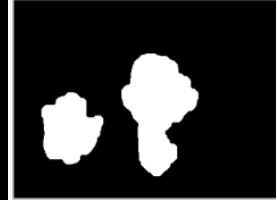

(b)

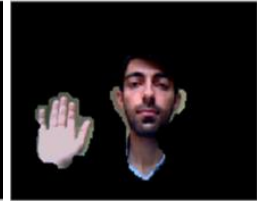

(c)
Fig. 4. Applying morphological operations on the segmented region. a) The binary segmented image, b) The resulting binary image, c) The resulting output

\section{Face Detection using AdaBoost}

After obtaining face region candidates, they must be verified to increase the accuracy. For this purpose, we use the Viola and Jones approach [30, 31]. Their approach includes three main steps. Firstly, a Haar-like feature set that later will be used by the detector is computed rapidly using Integral Image. Then we use the AdaBoost algorithm in order to select a small number of important features from the whole set to yield extremely efficient classifiers. Finally, with combining these classifiers as a cascade, we can quickly reject the background regions, while still more computation will be done on the remaining face-like regions by the next stages of the cascaded classifiers.

Actually, AdaBoost is an algorithm to create a powerful classifier from linear combination of the weaker classifiers. A set of Haar-like features is given as input to the cascade classifier. Each weak classifier implements a simple thresholding function on one of the features. With such a large set of weak classifiers, AdaBoost algorithm will select a small number of these weak classifiers and merge them into a classifier to decide whether an image is a face or non-face.

\section{FEATURE EXTRACTION AND FACE RECOGNITION}

At this stage after the detecting face, the detected peoples should be recognized. For this purpose, the feature extraction process should leave behind. This means, a series of features from the obtained faces should be extracted. The overview of the proposed feature extraction and face recognition system in Fig. 5 is shown.

Duties of the main blocks of this architecture are:

- Acquisition module: In fact this is the entry point to the face recognition process and provides the desired face image to the system.

- Pre-processing module: In this module, face images are normalized. These pre-processing steps can be used as normalization of image size, histogram matching, normalization of the rotation, normalization of light conditions, and etc. The most important of this pre-processing is normalization of the image size. This is usually done on the resulting image of the face detection process to change to the default size. This is the same image size that face detection system works with it.

- Feature extraction module: Here the normalized face image is sent to the feature extraction module in order to find the key features that are used for classification. In other words, this module is responsible for creating a feature vector that represents well the face image.

- Classification module: In this module using a template classifier, features extracted from the face images are compared with those faces that has been stored in database. After this comparison, the face image is recognized.

- Database: Classification module uses directly the face database.

\section{A. PCA Dimension Reduction}

Like many applications, the number of variable in the dataset needs to be reduced due to the existence of irrelevant, noisy and redundant information which has been proved to be the detrimental element leading to the inaccuracies in classification performance. Moreover, as the number of features for classification increases, the number of training samples required for statistical modeling and/or learning system grows exponentially. PCA is one of the methods that is used for feature extraction and reduction. The aim of this method is to find orthonormal vectors that have the most effect of the data. We can reduce the dimensions of the image data by eliminating the vectors with less effect. Although this may cause some loss of the image data [12, 32, 33]. 


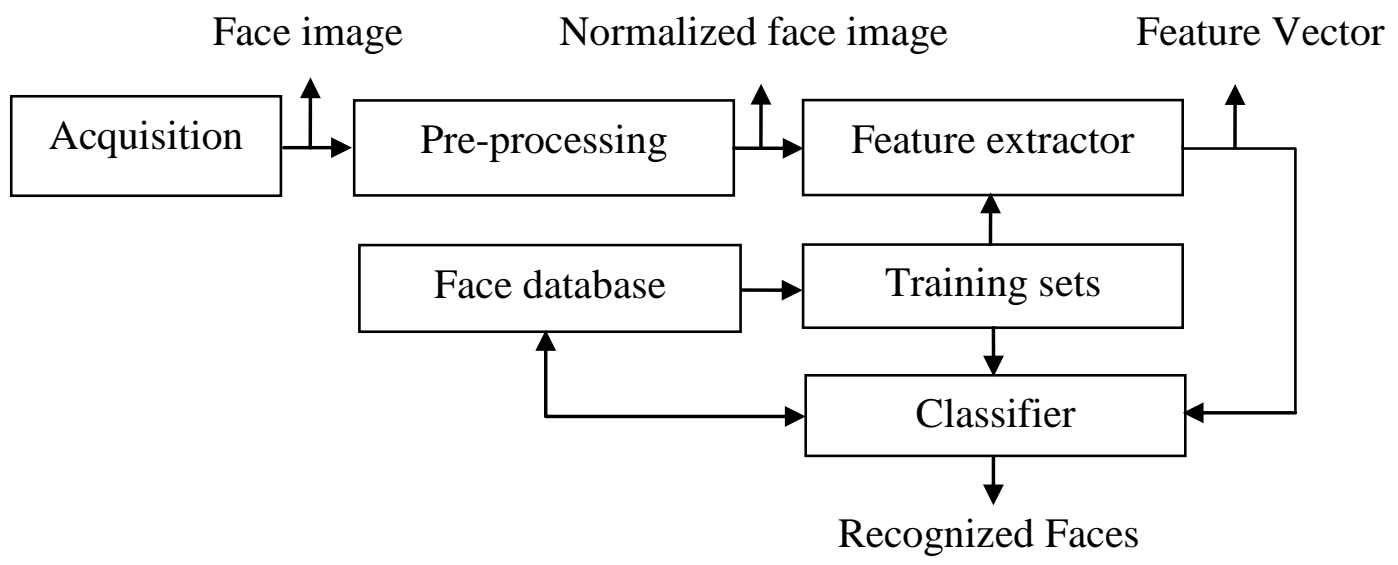

Fig. 5. Overview of the feature extraction and face recognition system

\section{B. LDA Feature Selection}

Undoubtedly, one of the most popular and experienced research trends in recent years is using different versions of the LDA in face recognition [32-34]. In this method, we seek to find $\mathrm{W}_{\mathrm{LDA}}$ vectors that can be able to separate different classes.

In this method, the first two-dimensional images with the dimensions of $\mathrm{a} \times \mathrm{b}$ are converted into vectors with $\mathrm{a}$ $\times$ b elemental. Two scatter matrixes inside the class, $S_{w}$, and outside the class, $S_{b}$, are used. The purpose is to produce a space as inside class scattering to be minimum and outside class scattering to be maximum.

Intuitively, if the data of each class are concentrated and the class masses are far from each other as far as possible, designing a learning machine for classifying the data will be easy and the learning machine will have a higher potential for classification of the data as well. Inside class scattering is an index for the concentration of the data in each class and the outside class scattering is an index for the amount of distances between the class masses.

An important point about the LDA method is that in the problems such as face recognition in which we are facing with large scale data and small training samples, $\mathrm{S}_{\mathrm{w}}$ may be close to a singular matrix, i.e. a matrix with zero determinant, that in case it will not be invertible. To fix this problem, first by using the PCA, image mapping is calculated in space with dimensions less than the image dimensions and then in space produced by PCA, LDA is applied [32, 33].

The main idea of PCA is to find the vectors which are best account for the distribution of the face images within the entire image space and selects the features important for class representation. LDA searches the directions for maximum discrimination of classes in addition to dimensionality reduction and selects the features that are most effective for class separability. Thus, first we apply
PCA to get the low dimensionality images which are ready to extract LDA features.

\section{SVM Classifications}

After extracting features, we classify samples by using the classifier. In machine learning, there are some conventional methods that have been extensively applied for multi-class classification problems. For example, the KNN classifier and artificial neural networks (e.g., Multilayer Perceptrons) can be mentioned. Each of these classifiers have their own advantages and disadvantages. Experimental results show that SVM classifier has the best performance compared to other classifiers [9]. SVM is originally designed for binary classification and performs pattern recognition between two classes [35]. The conventional way to extension of SVM to multi-class scenario is decomposing an $\mathrm{K}$-class problem into a series of two-class problems and constructing several binary classifiers. Some of them are: one-against-one and oneagainst-all.

For most applications, the one-against-one approach is more computationally intensive since the results of more SVM pairs must be computed. Regarding the training effort, the one-against-all approach is preferable since only K SVMs have to be trained in comparison with $\mathrm{K}$ ( $\mathrm{K}$ - 1)/ 2 SVMs in the one-against-one approach. The runtime complexity of the two strategies is similar: The oneagainst-all approach requires the evaluation of K SVMs, and the one-against-one approach requires the evaluation of $(K-1)$ SVMs. Due to this consideration, since the number of classes in face recognition can be rather large we have applied the one-against-all approach where the number of SVMs is linear with the number of classes.

\section{PROPOSED METHOD}

You can see the overall flowchart of the proposed system in Fig. 6. 


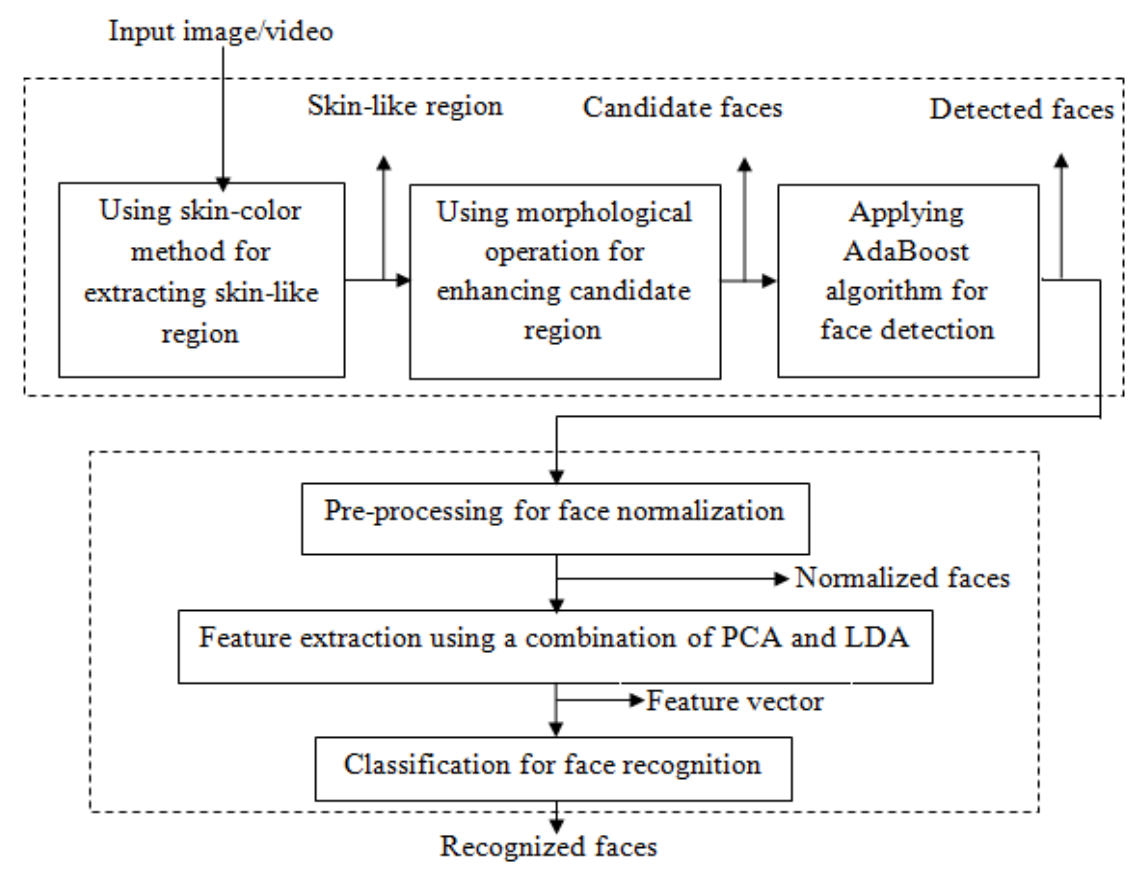

Fig. 6. Overall flowchart of the proposed system

First, for input video, the skin-like regions are extracted using skin-color method. To further improve the performance of the face detection part and to ensure higher accuracy, we applied a series of morphological operations. Then in the next step we extract the face from the candidate regions using the AdaBoost algorithm. This method is enough fast and suitable for video processing and takes advantage of both skin-color and AdaBoost methods. Employing skin-color segmentation algorithm, before the AdaBoost, will reduce the search area. Thus, we can reduce error detection rate and increase correct detection rate and also processing speed. Following this, a series of features from the detected face is extracted to recognize them. For this purpose, initially feature space dimensions reduced using the PCA method. Next, the desired features are extracted by the LDA method. With this operation we use the benefits of both above analysis. Finally, face recognition is done by applying the KNN or SVM classifiers.

\section{EXPERIMENTAL RESULT}

\section{A. Experimental Results of the Face Detection Algorithm}

For the face detection phase, two scenarios have been implemented.

Experimental results of the first scenario in which we use the Skin-color method in combination with the AdaBoost algorithm, show that the system is able to detect human faces in different lighting conditions, scales, poses, and skin color of different races. Fig. 7 shows some of the outcomes of the proposed method.

To test the face detection system, we used a series of
IBTD database images from UCSB Learning-Based Multimedia Lab, that includes images collected from the Internet. Using the skin color segmentation before the AdaBoost algorithm, reduces the searching region. As a result, not only the false positive rate reduces, but also the true positive rate and the processing speed can be increased. These results are shown in Table 1 and Table 2.

In Fig. 8 see some examples of the results of the algorithm.

Fig. 9 shows some examples of the results of the second scenario on different frames in which we use a combination of the Background Differencing method with the Skin-Color and AdaBoost algorithm.

The results of this scenario are shown in Table 3.

In the first scenario, with regard to this problem that constant background is not necessary, therefore, makes its application more general. However, since in many real applications, camera and the background are usually fixed, the second scenario can be employed in which we use the method of background differencing that is appropriate for video and real-time applications.
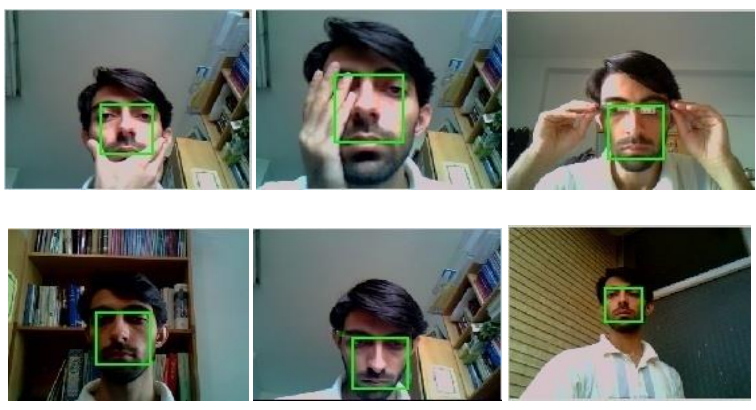

Fig. 7. Experimental results of different conditions 
Table 1. Results of Different Face Detection Methods

\begin{tabular}{|c|c|c|c|c|c|}
\hline Detection method & Face number & Hits & Misses & False alarms & Precision $(\%)$ \\
\hline Skin color & 325 & 288 & 37 & 26 & 88.6 \\
\hline AdaBoost & 325 & 271 & 54 & 45 & 83.4 \\
\hline $\begin{array}{c}\text { Skin color } \\
+ \text { AdaBoost }\end{array}$ & 325 & 306 & 19 & 15 & 94.2 \\
\hline
\end{tabular}

Table 2. Execution Time of Different Methods

\begin{tabular}{|c|c|c|}
\hline Detection method & AdaBoost & Skin color+AdaBoost \\
\hline Execution time $(\mathrm{ms})$ & 850 & 230 \\
\hline
\end{tabular}
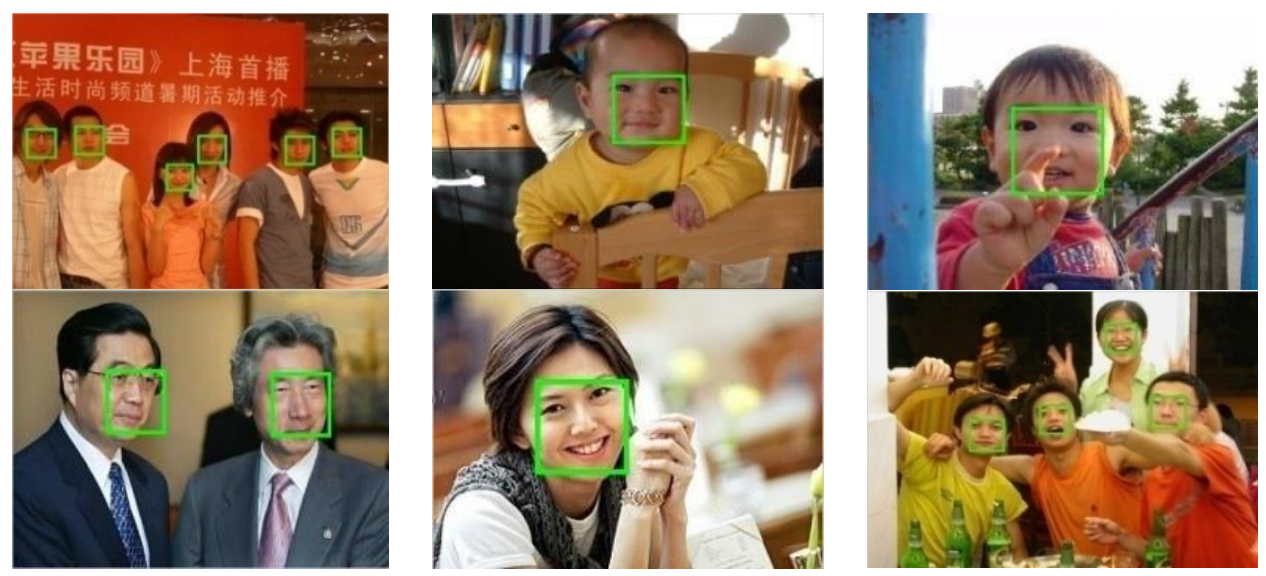

Fig. 8. Experimental results
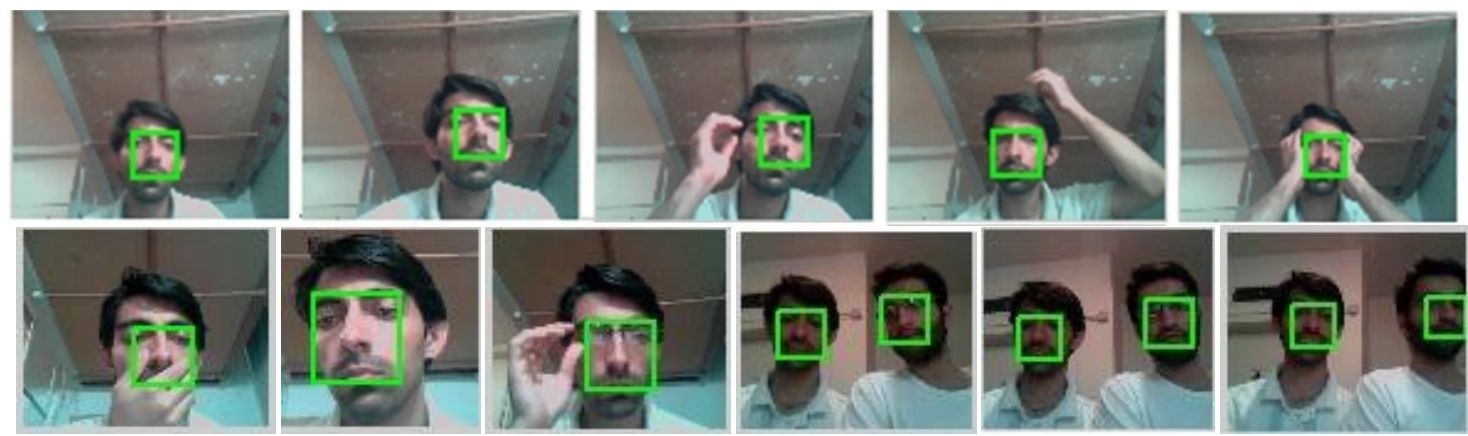

Fig. 9. the results of the second scenario applied on a sample video

Table 3. Results of comparison with other proposed methods

\begin{tabular}{|c|c|c|c|c|c|c|}
\hline $\begin{array}{c}\text { Detection } \\
\text { method }\end{array}$ & $\begin{array}{c}\text { Frame } \\
\text { number }\end{array}$ & $\begin{array}{c}\text { Face } \\
\text { number }\end{array}$ & hits & misses & $\begin{array}{c}\text { False } \\
\text { alarms }\end{array}$ & Precision $(\%)$ \\
\hline Skin color & 185 & 236 & 207 & 29 & 20 & 87.7 \\
\hline AdaBoost & 185 & 236 & 196 & 40 & 32 & 83.1 \\
\hline $\begin{array}{c}\text { Proposed } \\
\text { method }\end{array}$ & 185 & 236 & 217 & 19 & 7 & 91.9 \\
\hline
\end{tabular}

\section{B. Experimental Results of the Face Recognition Algorithm}

In the phase of feature extraction and face recognition, the following scenarios are considered:

- In the first scenario, the PCA method is used for feature extraction, then we do face recognition using KNN or SVM classifiers.
- In the second scenario, the LDA method is used for feature extraction, then we do face recognition using KNN or SVM classifiers.

- In the third scenario, firstly the dimensions of feature space is reduced using the PCA, and then using the LDA the desired features are extracted. Eventually, we do face recognition using KNN or SVM classifiers. 
- In the fourth scenario, a series of features are extracted by employing Gabor Wavelet filters. Next, using PCA and LDA methods dimensions of feature space are reduced and the desired characteristics are extracted. Finally, face recognition is done using $\mathrm{KNN}$ or SVM classifiers.

We used ORL database to evaluate and test the recognition part of our system. The ORL database contains 400 images corresponding to 40 subjects. The face database has variations in pose, size, time, facial expression and facial detail. Each image includes $112 \times 92$ pixels and has 256 gray levels. You can see some sample images of this database in Fig. 10.

We divide ten images of each person existing in the database into two groups, which means we choose the five images of each person as the training samples and the remaining five images as the testing samples of each person. So, totally the sum of training samples are 200 images and testing samples are 200 images. Table 4 shows the results of applying the different scenarios.

According to the Table 4, applying Gabor + PCA + LDA method has the best recognition rates. But Because of work on video, the system should be fast as far as possible, therefore, it is better to eliminate the feature extraction by Gabor and we use only the combination of PCA with LDA. Consequently, for our purpose the third scenario is chosen. Fig. 11 shows some example results of the third scenario on the ORL database. First row contains images that should be recognized and the second row shows images that have been retrieved from the database.

\section{Experimental Results of the system}

By applying the combination of two parts of the face detection and recognition on the videos that include different lighting conditions with different backgrounds, consecutively the best recognition rate is obtained. You can see the results of total proposed system in Table 5 .

In Fig. 12, some example results of applying the described method are observed. The first row shows the original frame. The second row shows the result of applying the skin-color algorithm, morphological operations, and the AdaBoost algorithm. In the third row, the normalized face image is shown. And ultimately in the fourth row, the face image retrieved from the database is displayed.

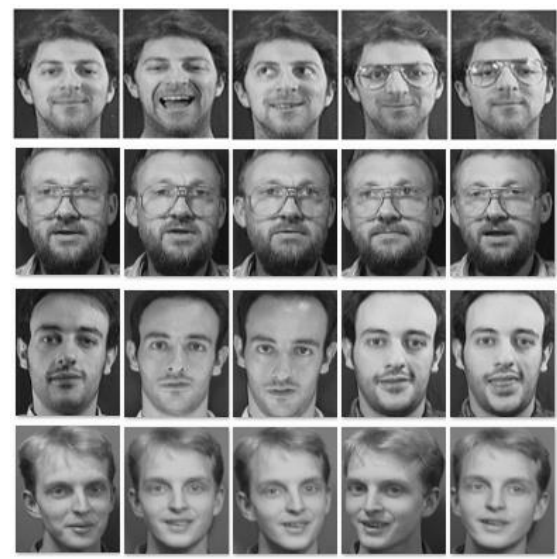

Fig. 10. A Group of Face Images in the ORL Database

Table 4. Results of Different Face Recognition Methods

\begin{tabular}{|c|c|c|c|c|}
\hline \multirow{2}{*}{ Method } & \multicolumn{4}{|c|}{ Recognition Rate (\%) } \\
\cline { 2 - 5 } Classifier & PCA & LDA & PCA+LDA & Gabor+PCA+LDA \\
\hline KNN & 87.5 & 91.2 & 93.5 & 96 \\
\hline SVM & 92.3 & 94.6 & 95 & 98 \\
\hline
\end{tabular}
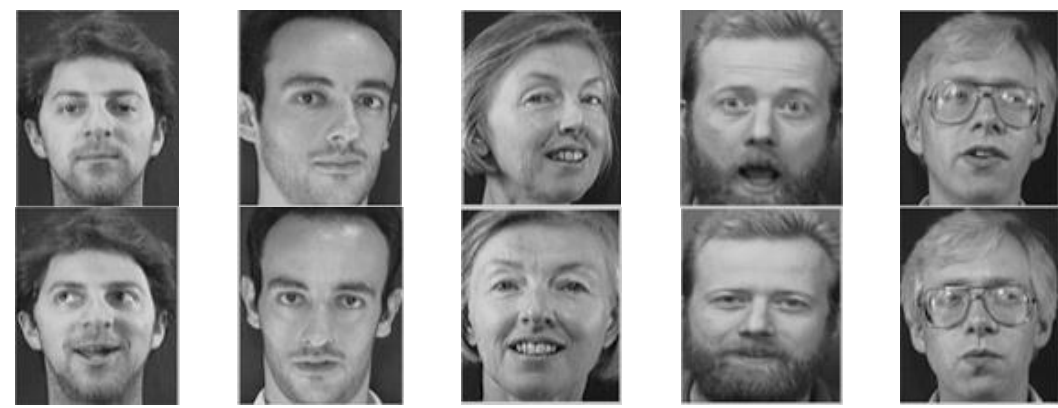

Fig. 11. Some results of the third scenario

Table 5. Results of Face Detection and Recognition System

\begin{tabular}{|c|c|c|c|c|}
\hline \multirow{2}{*}{ Method } & \multicolumn{3}{|c|}{ Detection and Recognition Rate (\%) } \\
\cline { 2 - 5 } & \multirow{2}{*}{ PCA } & LDA & PCA+LDA & Gabor+PCA+LDA \\
\hline KNN & 82 & 86 & 87.5 & 89.5 \\
\hline SVM & 85.5 & 88 & 89.5 & 93.5 \\
\hline
\end{tabular}




\section{CONCLUSION}

In this paper, we proposed a robust system for face detection and recognition. For the face detection phase, two scenarios have been implemented. In the first scenario, we used a combination of skin color segmentation and AdaBoost algorithm to make a rational trade off between the time complexity and accuracy and develop a high performance face detection algorithm. This leads to a serious reduction in the false positive rate and growth in the true positive rate and processing speed concurrently.

In the second scenario, assuming that the camera and the background are fixed, first, using the Background Differencing method we isolate the foreground region and then apply the Skin-Color algorithm on it and next, we take advantage of the AdaBoost algorithm.

Also, the face recognition part was developed as a combination of three stages: dimension reduction using PCA, feature selection using LDA, and K-NN or SVMbased classification. We employed these algorithms to construct an efficient face recognition system with a high recognition rate.

Simulation results show that the system is able to detect human faces in different lighting conditions, scales, poses, and various skin colors. Moreover, it also has the ability of optimal feature extraction and efficient face classification. Getting a high recognition rate of $93.5 \%$ demonstrated an improvement compared to the other known face recognition methods. The new face recognition algorithm can be used in many areas such as security applications.
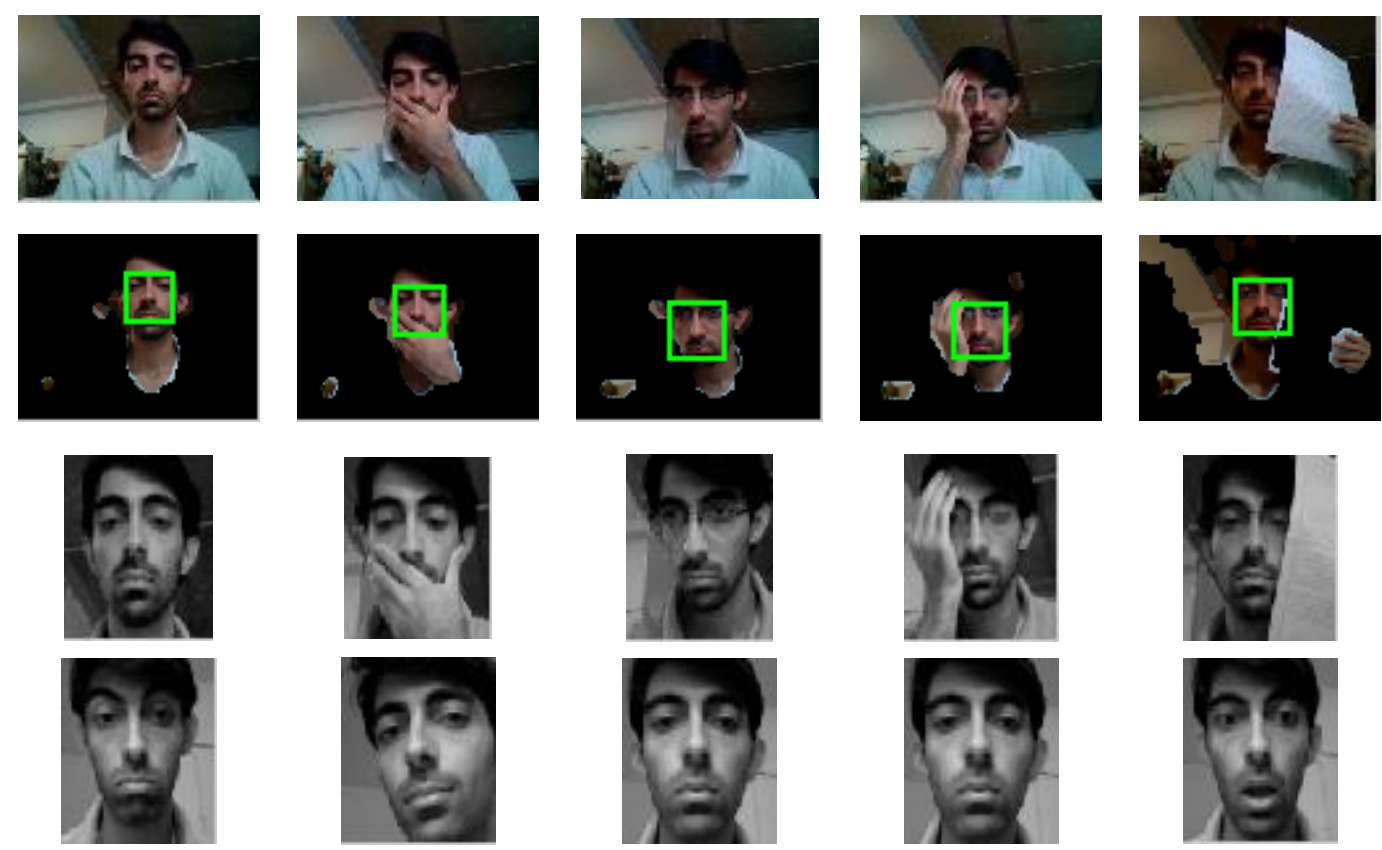

Fig. 12. Some examples of the implemented systems

\section{REFERENCES}

[1] M. Md. Maruf, P. Padma Polash, I. Md. Wahedul, and R. Siamak, "A Real-Time Face Recognition Approach from Video Sequence using Skin Color Model and Eigenface Method," in Electrical and Computer Engineering, 2006. CCECE '06. Canadian Conference on, 2006, pp. 21812185.

[2] E. Hjelmås and B. K. Low, "Face Detection: A Survey," Computer Vision and Image Understanding, vol. 83, pp. 236-274, 2001.

[3] Y. Ming-Hsuan and N. Ahuja, "Detecting human faces in color images," in Image Processing 98, 1998, pp. 127-130 vol.1.

[4] W. Zhao, R. Chellappa, P. J. Phillips, and A. Rosenfeld, "Face recognition: A literature survey," ACM Comput. Surv., vol. 35, pp. 399-458, 2003.

[5] H. Han-Pang and L. Chun-Ting, "Multi-CAMSHIFT for Multi-View Faces Tracking and Recognition," in Robotics and Biomimetics, 2006. ROBIO '06. IEEE International Conference on, 2006, pp. 1334-1339.
[6] Y. Ming-Hsuan, D. J. Kriegman, and N. Ahuja, "Detecting faces in images: a survey," Pattern Analysis and Machine Intelligence, IEEE Transactions on, vol. 24, pp. 34-58, 2002.

[7] J. Ruiz-del-Solar and P. Navarrete, "Eigenspace-based face recognition: a comparative study of different approaches," Systems, Man, and Cybernetics, Part C: Applications and Reviews, IEEE Transactions on, vol. 35, pp. 315-325, 2005.

[8] Y. Ben Jemaa and S. Khanfir, "Automatic Gabor Features Extraction for Face Recognition using Neural Networks," in Image Processing Theory, Tools and Applications, 2008. IPTA 2008. First Workshops on, 2008, pp. 1-6.

[9] M. O. Faruqe and M. Al Mehedi Hasan, "Face recognition using PCA and SVM," in Anti-counterfeiting, Security, and Identification in Communication, 2009. ASID 2009. 3rd International Conference on, 2009, pp. 97-101.

[10] X. Le Ha and S. Nitsuwat, "Face recognition in video, a combination of eigenface and adaptive skin-color model," in Intelligent and Advanced Systems, 2007. ICIAS 2007. International Conference on, 2007, pp. 742-747. 
[11] H. Sahoolizadeh and Y. A. Ghassabeh, "Face recognition using eigen-faces, fisher-faces and neural networks," in Cybernetic Intelligent Systems, 2008. CIS 2008. 7th IEEE International Conference on, 2008, pp. 1-6.

[12] M. A. Turk and A. P. Pentland, "Face recognition using eigenfaces," in Computer Vision and Pattern Recognition, 1991. Proceedings CVPR '91., IEEE Computer Society Conference on, 1991, pp. 586-591.

[13] K. Delac, M. Grgic, and S. Grgic, "Statistics in face recognition: analyzing probability distributions of PCA, ICA and LDA performance results," in Image and Signal Processing and Analysis, 2005. ISPA 2005. Proceedings of the 4th International Symposium on, 2005, pp. 289-294.

[14] L. Juwei, K. N. Plataniotis, and A. N. Venetsanopoulos, "Face recognition using LDA-based algorithms," Neural Networks, IEEE Transactions on, vol. 14, pp. 195-200, 2003.

[15] J. Lu, K. N. Plataniotis, and A. N. Venetsanopoulos, "Regularization studies on LDA for face recognition," in Image Processing, 2004. ICIP '04. 2004 International Conference on, 2004, pp. 63-66 Vol. 1.

[16] L. Weisheng and C. Wanli, "Face Recognition Based on Adaptively Weighted Gabor-LDA," in Natural Computation, 2008. ICNC '08. Fourth International Conference on, 2008, pp. 130-134.

[17] L. Chengjun and H. Wechsler, "Independent component analysis of Gabor features for face recognition," Neural Networks, IEEE Transactions on, vol. 14, pp. 919-928, 2003.

[18] J. G. Daugman, "Complete discrete 2-D Gabor transforms by neural networks for image analysis and compression," Acoustics, Speech and Signal Processing, IEEE Transactions on, vol. 36, pp. 1169-1179, 1988.

[19] L. Tai Sing, "Image representation using 2D Gabor wavelets," Pattern Analysis and Machine Intelligence, IEEE Transactions on, vol. 18, pp. 959-971, 1996.

[20] W. Meihua, J. Hong, and L. Ying, "Face recognition based on DWT/DCT and SVM," in Computer Application and System Modeling (ICCASM), 2010 International Conference on, 2010, pp. V3-507-V3-510.

[21] E. Meng Joo, W. Chen, and W. Shiqian, "High-speed face recognition based on discrete cosine transform and RBF neural networks," Neural Networks, IEEE Transactions on, vol. 16, pp. 679-691, 2005.

[22] J. Xiao-Yuan and D. Zhang, "A face and palmprint recognition approach based on discriminant DCT feature extraction," Systems, Man, and Cybernetics, Part B: Cybernetics, IEEE Transactions on, vol. 34, pp. 2405-2415, 2004.

[23] A. M. Martinez and A. C. Kak, "PCA versus LDA," Pattern Analysis and Machine Intelligence, IEEE Transactions on, vol. 23, pp. 228-233, 2001.

[24] L. Bing, Z. Yun, and P. Yun-Hong, "Face recognition based on wavelet transform and SVM," in Information Acquisition, 2005 IEEE International Conference on, 2005 , pp. 373-377.

[25] C.-j. Chen, Y.-z. Zhan, and C.-j. Wen, "Hierarchical Face Recognition Based on SVDD and SVM," in Environmental Science and Information Application Technology, 2009. ESIAT 2009. International Conference on, 2009, pp. 692695.

[26] H. Cheng-Ho and W. Jhing-Fa, "SVM-based one-againstmany algorithm for liveness face authentication," in Systems, Man and Cybernetics, 2008. SMC 2008. IEEE International Conference on, 2008, pp. 744-748.

[27] M. H. Mousavi, K. Faez, and A. Asghari, "Three Dimensional Face Recognition Using SVM Classifier," in
Computer and Information Science, 2008. ICIS 08. Seventh IEEE/ACIS International Conference on, 2008, pp. 208213.

[28] P. Kakumanu, S. Makrogiannis, and N. Bourbakis, "A survey of skin-color modeling and detection methods," Pattern Recognition, vol. 40, pp. 1106-1122, 2007.

[29] J. M. Chaves-Gonzalez, M. A. Vega-Rodriguez, J. A. Ǵmez-Pulido, and J. M. Sanchez-Pérez, "Detecting skin in face recognition systems: A colour spaces study," Digital Signal Processing, vol. 20, pp. 806-823, 2010.

[30] P. Viola and M. Jones, "Rapid object detection using a boosted cascade of simple features," in Computer Vision and Pattern Recognition, 2001. CVPR 2001. Proceedings of the 2001 IEEE Computer Society Conference on, 2001, pp. I-511-I-518 vol.1.

[31] P. Viola and M. Jones, "Robust Real-Time Face Detection," Int. J. Comput. Vision, vol. 57, pp. 137-154, 2004.

[32] P. N. Belhumeur, J. P. Hespanha, and D. J. Kriegman, "Eigenfaces vs. Fisherfaces: recognition using class specific linear projection," Pattern Analysis and Machine Intelligence, IEEE Transactions on, vol. 19, pp. 711-720, 1997.

[33] W. Zhao, R. Chellappa, and A. Krishnaswamy, "Discriminant analysis of principal components for face recognition," in Automatic Face and Gesture Recognition, 1998. Proceedings. Third IEEE International Conference on, 1998, pp. 336-341.

[34] E. Kamran and C. Rama, "Discriminant Analysis for Recognition of Human Face Images (Invited Paper)," in Proceedings of the First International Conference on Audio- and Video-Based Biometric Person Authentication, Springer-Verlag, 1997.

[35] H. Chih-Wei and L. Chih-Jen, "A comparison of methods for multiclass support vector machines," Neural Networks, IEEE Transactions on, vol. 13, pp. 415-425, 2002.

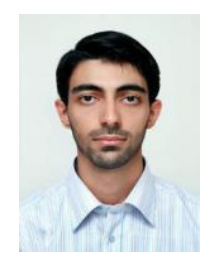

Alireza Tofighi, male, received the BS and MS degrees in computer engineering from Ferdowsi University of Mashhad and University of Isfahan, Iran in 2008 and 2011, respectively. His research interests include machine vision, image processing, signal processing and pattern recognition.

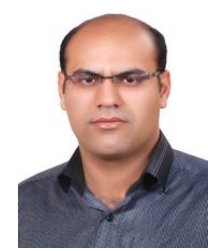

Nima Khairdoost, male, received the BS and MS degrees in computer engineering from Ferdowsi University of Mashhad and University of Isfahan, Iran in 2008 and 2011, respectively. He currently is a lecturer in the Engineering Department of Tabaran Institute of Higher Education, Mashhad, Iran. His research interests include image processing, machine vision and pattern recognition as well as evolutionary algorithms.

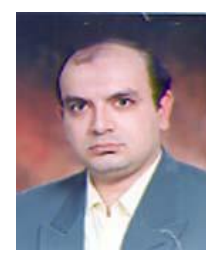

S. Amirhassan Monadjemi, male, born 1968 in Isfahan, Iran. He received his BS degree at computer hardware engineering from Isfahan University of Tech., in 1991, his MS degree at computer engineering, machine intelligence and robotics from Shiraz University, Shiraz, in 1994 and his $\mathrm{PhD}$ degree in computer science, image processing and pattern recognition from 
University of Bristol, Bristol, UK. in 2004. He is now working as associate professor at the Department of Computer Engineering, Faculty of Engineering, University of Isfahan, Isfahan, Iran. His research interests include image processing, computer vision and pattern recognition, computer aided learning and physical detection and elimination of viruses.

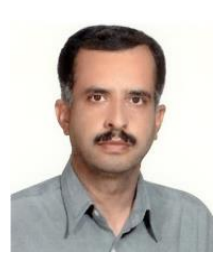

Kamal Jamshidi, male, received the MS and $\mathrm{PhD}$ degrees in electrical engineering from Anna University of India in 1990 and I.I.T University of India in 2003, respectively. He currently is an associate professor in the Engineering Department of University of Isfahan. His research interests include wireless sensor network and vehicular ad hoc networks as well as fuzzy systems and microprocessor based systems.

How to cite this paper: Alireza Tofighi, Nima Khairdoost, S. Amirhassan Monadjemi, Kamal Jamshidi,"A Robust Face Recognition System in Image and Video", IJIGSP, vol.6, no.8, pp.1-11, 2014.DOI: 10.5815/ijigsp.2014.08.01 